\title{
Source formulation for electron-impact ionization for fluid plasma simulations
}

\author{
Müller, S.H.; Holland, C.; Tynan, G.R.; Yu, J.H.; Naulin, Volker
}

Published in:

Plasma Physics and Controlled Fusion

Link to article, DOI:

$10.1088 / 0741-3335 / 51 / 10 / 105014$

Publication date:

2009

Link back to DTU Orbit

Citation (APA):

Müller, S. H., Holland, C., Tynan, G. R., Yu, J. H., \& Naulin, V. (2009). Source formulation for electron-impact ionization for fluid plasma simulations. Plasma Physics and Controlled Fusion, 51(10), 105014.

https://doi.org/10.1088/0741-3335/51/10/105014

\section{General rights}

Copyright and moral rights for the publications made accessible in the public portal are retained by the authors and/or other copyright owners and it is a condition of accessing publications that users recognise and abide by the legal requirements associated with these rights.

- Users may download and print one copy of any publication from the public portal for the purpose of private study or research.

- You may not further distribute the material or use it for any profit-making activity or commercial gain

- You may freely distribute the URL identifying the publication in the public portal

If you believe that this document breaches copyright please contact us providing details, and we will remove access to the work immediately and investigate your claim 


\title{
Source formulation for electron-impact ionization for fluid plasma simulations
}

\author{
S. H. Müller, ${ }^{1}$ C. Holland, ${ }^{1}$ G. R. Tynan, ${ }^{1}$ J. H. Yu ${ }^{1}$ and V. Naulin ${ }^{2}$ \\ ${ }^{1}$ Center for Energy Research - University of California, San Diego, \\ 9500 Gilman Drive, M/C 0417, La Jolla, CA-92093, USA \\ ${ }^{2}$ Association EURATOM, Risø National Laboratory, Technical University of Denmark, \\ Department of Optics and Plasma Research Dynamics, \\ P.O. Box 49, DK-4000 Roskilde, Denmark
}

(Dated: August 6, 2009)

\begin{abstract}
The derivation of the correct functional form of source terms in plasma fluid theory is revisited. The relation between the fluid source terms and atomic physics differential cross sections is established for particle-impact ionization. It is shown that the interface between atomic and plasma physics is completely described by three scalar functions of the incident particle energy. These are the total cross section and the newly introduced forward momentum and energy functions, which are properties of the differential cross sections only. For electron-impact ionization, the Binary-Encounter-Bethe (BEB) and Binary-Encounter-Dipole (BED) models [Y.-K. Kim and M. E. Rudd, Phys. Rev. A, 50 (1994) 3954] are used to calculate these functions analytically, yielding expressions that both accurately capture the physics and can be efficiently evaluated within fluid simulation codes. The source terms explain the observed electron temperature regimes in a wide variety of basic plasma physics experiments, including the trends across different gases.
\end{abstract}




\section{INTRODUCTION}

Numerical simulations of plasma turbulence and transport in magnetic confinement fusion devices can be divided into three main groups: (i) Gyrokinetic simulations of the plasma core; (ii) transport codes using reduced models to simulate entire discharges; (iii) fluid simulations of the plasma edge and Scrape-Off-Layer (SOL). The treatment of sources and sinks has a very different status in each of these code families.

Gyrokinetic codes initially focused on the computation of heat fluxes in the plasma core for prescribed profiles. As the simulated time span was much shorter than the confinement time, the effect of sources and sinks could be neglected. However, gyrokinetic codes have since evolved to address steady-state turbulence, for which sources and dissipation needed to be added. At present, the source terms are chosen $a d$-hoc, either by adding an adjustable right-hand side that has the property to enforce a certain distribution function at certain locations with a certain time constant [1-3], or by locally rescaling the energy of marker particles [4]. Significant attention is devoted merely to the question whether a true statistical steady-state is achieved in such simulations [2].

While the realistic modeling of sources in gyrokinetic codes is in its infancy, the opposite is true for the family of transport codes such as ASTRA [5], ONETWO [6] and TRANSP [7] for the tokamak core and B2-EIRENE [8], EDGE2D-NIMBUS [9] and UEDGE [10] for the SOL. The reduced description of the plasma physics in these codes makes the simulation of entire discharges in actual experimental devices tractable. Transport codes make extensive use of modules libraries [11] to simulate plasma heating schemes and plasma-neutral interaction. These comprise ray-tracing codes such as TORAY-GA [12] to calculate the power deposition and current-drive by RF waves [13], Monte-Carlo codes such as NUBEAM [14] to calculate the power deposition and momentum transfer of fast neutrals/ions from neutral beam injection [15], and Monte-Carlo codes implementing collisional-radiative models of the SOL, such as EIRENE [8], NIMBUS [9] and DEGAS2 [16]. These codes generally include a fairly complete and sophisticated description of atomic physical processes, but, to our knowledge, have not taken the step to make use of differential cross sections to calculate sources and sinks kinetically.

While transport codes employ macroscopic models for the turbulent transport, edge turbulence codes, such as BOUT [17] and ESEL [18], follow the fluid turbulent dynamics 
self-consistently. For the tokamak edge and SOL, these simulations generally adopt a fluxdriven framework, where the sources lie outside of the simulated domain, thus avoiding the problem of dealing with volume sources and sinks to a certain extent [18]. However, it has been realized that basic plasma physics experiments, such as the torsatron TJ-K [19], the linear devices CSDX [20] and VINETA [21], and the simple magnetized tori TORPEX [22] and HELIMAK [23], provide ideal testbeds for fluid turbulence simulation codes, and efforts have begun to validate the models behind the codes against these experiments [24-31]. In these devices, volume sources due to ionization via a fast electron population are dominant.

Earlier simulations prescribed the time-average profiles [24-26, 28, 29], in which case no explicit sources are necessary. To allow for self-consistently evolving profiles, newer simulations implemented $a d-h o c$ source terms [27, 30]. While reference [27] used an isothermal model, in which only a density source needed to be assumed, reference [30] allowed for a fully turbulent temperature evolution, making both a density and an energy source necessary. Implementing ad-hoc choices for the source terms, simulations of the TORPEX device showed a strong dependence of the turbulence dynamics on the freely adjustable temperature source $S_{T}$ [30]. In particular, an 'H-mode' like regime was predicted, in which the emergence of blobs [32] would be suppressed. However, this regime could not be observed experimentally [33]. Attempts to estimate $S_{T}$ from experimental data or to relate it to the experimentally more accessible density source $S_{n}$ have failed, revealing instead more fundamental problems with the formulation of sources in [30]. This highlights the necessity to revisit the problem of self-consistent source formulation for fluid turbulence codes - in analogy to the evolution of transport codes.

Although much can be learned and taken from the development of transport codes, the requirements to implement sources in turbulence codes, which have to use a much smaller time step, are not exactly compatible. For example, time-consuming Monte-Carlo approaches to determine ionization rates are not so attractive. One would also not want to lose the advantage of applying analytical theory, facilitated by the generally simpler geometry, such that a deterministic and, if possible, analytical description of sources would be favorable. However, no compromises should be made on the consistency of density, momentum and energy sources, as the transfer of energy between the different forms is an aspect of primary importance in turbulence studies.

The goal of this paper is to provide such a practical, self-consistent description of sources 
for fluid turbulence simulations, focusing on electron-impact ionization. The derivation of self-consistent source terms in fluid plasma theory is revisited (section II) and their relation to atomic physics differential cross sections is established (section III). In section IV, a fairly recent atomic physics theory is applied to analytically calculate self-consistent density, momentum and energy source terms, which can be evaluated efficiently within fluid turbulence codes. In section $\mathrm{V}$, we discuss the implications of these source terms, which accurately explain the experimentally observed electron temperature regimes in a wide range of basic plasma physics experiments, including the trends across different gases.

\section{FUNCTIONAL FORM OF FLUID SOURCE TERMS}

The starting point to derive self-consistent fluid source terms for each species with mass $m$ and charge $q$ is the kinetic equation augmented by a source term

$$
\frac{\partial f}{\partial t}+\mathbf{v} \cdot \nabla f+\frac{q}{m}(\mathbf{E}+\mathbf{v} \times \mathbf{B}) \cdot \nabla_{v} f=\mathcal{C}\{f\}+\mathcal{S}(\mathbf{x}, \mathbf{v}, t)
$$

where $\mathcal{C}\{f\}$ is the collision operator, accounting for all elastic processes, and $\mathcal{S}(\mathbf{x}, \mathbf{v}, t)$ is a completely general kinetic source term, which in principle accounts for all inelastic atomic and molecular processes in the plasma, such as ionization, recombination and charge exchange, as well as absorption and emission of radiation. Since the following procedure is only partially described in the literature [8, 34-36], the full derivation shall be given here. Taking the moments $\left\{1, m \mathbf{v}, \frac{m}{2} v^{2}\right\}$ of (1) leads to the fluid equations in the laboratory frame (subscript " $L$ "), also called conservative form:

$$
\begin{aligned}
\frac{\partial n}{\partial t}+\nabla \cdot(n \mathbf{V}) & =S_{n} \\
m \frac{\partial(n \mathbf{V})}{\partial t}+\nabla p+\nabla \cdot(\stackrel{\leftrightarrow}{\pi}+m n \mathbf{V} \mathbf{V})-q n(\mathbf{E}+\mathbf{V} \times \mathbf{B}) & =\mathbf{R}+\mathbf{S}_{k, L} \\
\frac{\partial}{\partial t}\left(\frac{3}{2} p+\frac{1}{2} m n V^{2}\right)+\nabla \cdot \mathbf{Q}-q n \mathbf{V} \cdot \mathbf{E} & =W_{L}+S_{\mathcal{E}, L}
\end{aligned}
$$

where

$$
\begin{array}{rlrl}
n & \equiv \int d^{3} v f, & n \mathbf{V} \equiv \int d^{3} v \mathbf{v} f \\
p & \equiv m \int d^{3} v w^{2} f, & \pi_{i j} & \equiv m \int d^{3} v\left(w_{i} w_{j}-w^{2} \delta_{i j}\right) f, \\
\mathbf{q} & \equiv \frac{m}{2} \int d^{3} v w^{2} \mathbf{w} f, & \mathbf{Q} & \equiv \mathbf{q}+\frac{5}{2} p \mathbf{V}+\stackrel{\leftrightarrow}{\pi} \cdot \mathbf{V}+\frac{1}{2} m n V^{2} \mathbf{V},
\end{array}
$$


where $\mathbf{w} \equiv \mathbf{v}-\mathbf{V}$, and

$$
\begin{aligned}
0 & =\int d^{3} v \mathcal{C}, & S_{n} & \equiv \int d^{3} v \mathcal{S}, \\
\mathbf{R} & \equiv m \int d^{3} v \mathbf{v} \mathcal{C}, & \mathbf{S}_{k, L} & \equiv m \int d^{3} v \mathbf{v} \mathcal{S}, \\
W_{L} & \equiv \frac{m}{2} \int d^{3} v v^{2} \mathcal{C}, & S_{\mathcal{E}, L} & \equiv \frac{m}{2} \int d^{3} v v^{2} \mathcal{S} .
\end{aligned}
$$

It is useful to define the average operator

$$
\langle\cdot\rangle \equiv \frac{\int d^{3} v \mathcal{S}(\mathbf{v})(\cdot)}{\int d^{3} v \mathcal{S}(\mathbf{v})}
$$

to highlight the relation of the momentum and energy sources to the particle source:

$$
\begin{aligned}
& \mathbf{S}_{k, L}=\langle\mathbf{k}\rangle S_{n}, \\
& S_{\mathcal{E}, L}=\langle\mathcal{E}\rangle S_{n},
\end{aligned}
$$

where $\mathbf{k} \equiv m \mathbf{v}$ and $\mathcal{E} \equiv \frac{m}{2} v^{2}$. The fluid equations in the plasma frame can be obtained either by repeatedly substituting the lower-order moment equations into the higher-order moment equations, or by calculating the moments $\left\{1, m \mathbf{w}, \frac{m}{2} w^{2}\right\}$ directly:

$$
\begin{aligned}
\frac{d n}{d t}+n \nabla \cdot \mathbf{V} & =S_{n}, \\
m n \frac{d \mathbf{V}}{d t}+\nabla p+\nabla \cdot \overleftrightarrow{\pi}-q n(\mathbf{E}+\mathbf{V} \times \mathbf{B}) & =\mathbf{R}+\mathbf{S}_{k}, \\
\frac{3}{2} \frac{d p}{d t}+\frac{5}{2} p \nabla \cdot \mathbf{V}+\overleftrightarrow{\pi}: \nabla \mathbf{V}+\nabla \cdot \mathbf{q} & =W+S_{\mathcal{E}},
\end{aligned}
$$

where $d / d t \equiv \partial / \partial t+\mathbf{V} \cdot \nabla, \overleftrightarrow{\pi}: \nabla \mathbf{V} \equiv \pi_{i j} \partial_{i} V_{j}, W \equiv W_{L}-\mathbf{V} \cdot \mathbf{R}$, and $\mathbf{S}_{k}$ and $S_{\mathcal{E}}$ are the sources in the plasma frame:

$$
\begin{aligned}
& \mathbf{S}_{k} \equiv \mathbf{S}_{k, L}-m \mathbf{V} S_{n}=(\langle\mathbf{k}\rangle-m \mathbf{V}) S_{n}, \\
& S_{\mathcal{E}} \equiv S_{\mathcal{E}, L}-\mathbf{V} \cdot \mathbf{S}_{k, L}+\frac{m}{2} V^{2} S_{n}=\left(\langle\mathcal{E}\rangle-\mathbf{V} \cdot\langle\mathbf{k}\rangle+\frac{m}{2} V^{2}\right) S_{n} .
\end{aligned}
$$

Note the contributions from the lower-order source moments that appear due to the transformation to the plasma frame.

If the momentum and energy equations are rewritten into equations for the velocity $\mathbf{V}$ and the temperature $T \equiv p / n$ of the form $\partial \mathbf{V} / \partial t+\ldots=\mathbf{S}_{V}$ and $\partial T / \partial t+\ldots=S_{T}$, the source terms take the form:

$$
\begin{aligned}
\mathbf{S}_{V}(n, \mathbf{V}) & \equiv\left(\frac{\langle\mathbf{k}\rangle}{m}-\mathbf{V}\right) \frac{S_{n}}{n}, \\
S_{T}(n, \mathbf{V}, T) & \equiv \frac{2}{3}\left(\langle\mathcal{E}\rangle-\mathbf{V} \cdot\langle\mathbf{k}\rangle+\frac{m}{2} V^{2}-\frac{3}{2} T\right) \frac{S_{n}}{n} .
\end{aligned}
$$


In cases where ambipolarity holds, the speed $V$ of electrons and ions is limited by $V \lesssim c_{s}$, where $c_{s} \equiv \sqrt{T_{e} / m_{i}}$ is the sound speed. Therefore, for electrons, the ratio between the energy in the directed motion to the thermal energy scales as $\left|\left(\mathbf{V}_{e} \cdot\left\langle\mathbf{k}_{e}\right\rangle-\frac{m_{e}}{2} V_{e}^{2}\right) /\left(\left\langle\mathcal{E}_{e}\right\rangle-\frac{3}{2} T_{e}\right)\right| \sim$ $c_{s}^{2} / v_{\text {the }}^{2}=m_{e} / m_{i} \ll 1$. In such a case, the electron temperature source term may be approximated as

$$
S_{T_{e}}\left(n_{e}, \mathbf{V}_{e}, T_{e}\right) \approx\left(\frac{2}{3}\left\langle\mathcal{E}_{e}\right\rangle-T_{e}\right) \frac{S_{n_{e}}}{n_{e}} .
$$

The sources $\mathbf{S}_{V}$ and $S_{T}$ act to equilibrate the plasma velocity and temperature to the average velocity and thermodynamic kinetic energy (in the plasma frame) of the newly generated particles, respectively. This happens at the same rate $S_{n} / n$ at which new particles are generated. It is obvious that $\mathbf{S}_{V}$ and $S_{T}$ dynamically assume both positive and negative values, depending on whether the current plasma velocity and temperature is lower or higher than the neutral values

$$
\mathbf{V}_{\infty} \equiv \frac{\langle\mathbf{k}\rangle}{m}, \quad T_{\infty} \equiv \frac{2}{3}\left(\langle\mathcal{E}\rangle-\frac{\langle\mathbf{k}\rangle^{2}}{2 m}\right) .
$$

As even the first few plasma particles are generated with the average momentum $\langle\mathbf{k}\rangle$ and average energy $\langle\mathcal{E}\rangle$, appropriate initial conditions for numerical simulations are $\mathbf{V}(0)=\mathbf{V}_{\infty}$ and $T(0)=T_{\infty}$. Isothermal models are compatible with the self-consistent source terms if $T=T_{\infty}$.

\section{A. Action of the sources}

In the following, we consider a few illustrative cases to understand the interdependent action of the density, velocity and temperature sources. We assume a homogeneous plasma $(\nabla \rightarrow 0)$, with no fields $(\mathbf{E}=0, \mathbf{B}=0)$ and no collisions $(\mathbf{R}=0, W=0)$. The fluid equations reduce to $\partial n / \partial t=S_{n}, \partial \mathbf{V} / \partial t=\mathbf{S}_{V}$ and $\partial T / \partial t=S_{T}$, or in conservative form

$$
\begin{aligned}
\frac{\partial n}{\partial t} & =S_{n}, \\
\frac{\partial(n \mathbf{V})}{\partial t} & =\frac{\langle\mathbf{k}\rangle}{m} \frac{\partial n}{\partial t}, \\
\frac{\partial}{\partial t}\left(\frac{3}{2} p+\frac{1}{2} m n V^{2}\right) & =\langle\mathcal{E}\rangle \frac{\partial n}{\partial t} .
\end{aligned}
$$



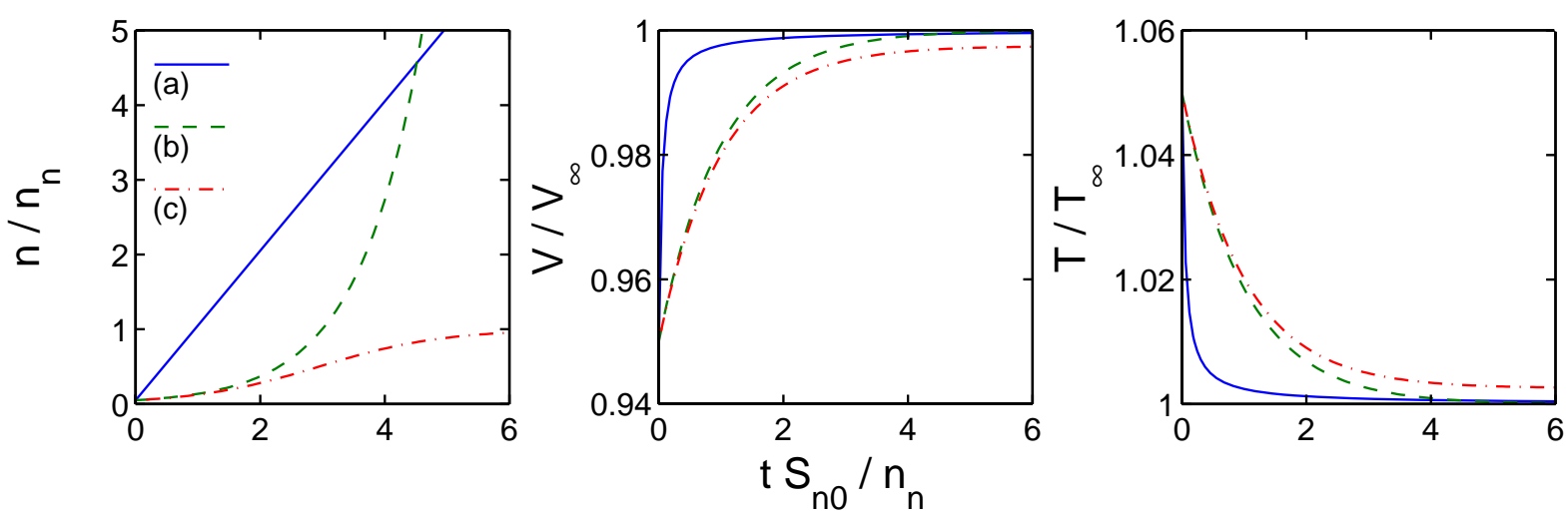

FIG. 1: Action of the source terms in Eq. 28 for density, velocity, and temperature, for $\langle\mathbf{k}\rangle^{2} /(2 m\langle\mathcal{E}\rangle)=0.2$.

Assuming that $\langle\mathbf{k}\rangle$ and $\langle\mathcal{E}\rangle$ are constant in time, the momentum and energy equations can be integrated regardless of the actual form of $S_{n}$ :

$$
\begin{aligned}
\mathbf{V}(t) & =\mathbf{V}_{0} \frac{n_{0}}{n}+\frac{\langle\mathbf{k}\rangle}{m}\left(1-\frac{n_{0}}{n}\right), \\
\frac{3}{2} T(t) & =\left(\frac{3}{2} T_{0}-\frac{m}{2}\left(V^{2}-V_{0}^{2}\right)\right) \frac{n_{0}}{n}+\left(\langle\mathcal{E}\rangle-\frac{m}{2} V^{2}\right)\left(1-\frac{n_{0}}{n}\right),
\end{aligned}
$$

where $n_{0} \equiv n(0), \mathbf{V}_{0} \equiv \mathbf{V}(0)$ and $T_{0} \equiv T(0)$ are the initial conditions. As $n$ gets much larger than $n_{0}$, the limits for velocity and temperature stay finite, and are given by $\mathbf{V}_{\infty}$ and $T_{\infty}$ as given in Eq. 22. The fact that $\mathbf{V}$ and $T$ do not grow without bounds reflects their true nature as intensive thermodynamic quantities, as opposed to momentum and energy, which are extensive thermodynamic variables.

We now consider three different forms for $S_{n}$ and their solution for $n(t)$ :

$$
\begin{aligned}
S_{n} & =S_{n 0}: & n(t) & =n_{0}+S_{n 0} t \\
S_{n} & =\frac{n}{n_{n}} S_{n 0}: & n(t) & =n_{0} \exp \left(\frac{S_{n 0}}{n_{n}} t\right), \\
S_{n} & =\frac{n}{n_{n}}\left(1-\frac{n}{n_{n}}\right) S_{n 0}: & n(t) & =\frac{n_{0}}{\frac{n_{0}}{n_{n}}+\left(1-\frac{n_{0}}{n_{n}}\right) \exp \left(-\frac{S_{n 0}}{n_{n}} t\right)},
\end{aligned}
$$

where $n_{n}$ is the density of neutral atoms. Case (a) represents the situation where the plasma production is decoupled from the plasma, such as plasma production by an independent beam. Cases (b) and (c) correspond to a situation where the plasma provides the electrons necessary for ionization, with case (c) accounting for the depletion of the target population 
as the number of ionized atoms increases. Figure 1 illustrates the action of the source terms in Eq. 28 on density, velocity and temperature for the case $\langle\mathbf{k}\rangle^{2} /(2 m\langle\mathcal{E}\rangle)=0.2$. The initial conditions are $n_{0}=0.05 n_{n}, V_{0}=0.95 V_{\infty}, T_{0}=1.05 T_{\infty}$, representing a case where the source acts to reduce the temperature. For the form of the density source in Eq. 28c, plasma production comes to a stop once all available neutrals are ionized $\left(n=n_{n}\right)$. As $n / n_{0}$ cannot grow without bounds in this case, the limits $V_{\infty}$ and $T_{\infty}$ are only approximately reached.

\section{RELATION TO DIFFERENTIAL CROSS SECTIONS}

So far, our analysis was completely general and consisted merely of algebraic manipulations of an arbitrary source term $\mathcal{S}(\mathbf{v})$ in the kinetic equation. We now turn to the formulation of source terms for a specific physical process, namely binary particle-impact ionization, in which a single incident particle strikes an atom/ion and frees a single electron. This includes electron-impact, ion-impact and photo-ionization, which are the generating processes for the vast majority of laboratory, astrophysical and fusion plasmas. The following discussion is, to the best of our knowledge, the first in plasma physics literature that applies differential atomic-physics cross sections to compute plasma density, momentum and energy sources self-consistently.

For the following treatment to remain valid for massless incident particles, and to facilitate cross-referencing with atomic physics literature, we will use distribution functions of the form $F(\mathcal{E}, \theta, \varphi)$ to describe the particle populations, where $\mathcal{E}$ is the particle energy and $(\theta, \varphi)$ are the angles of the momentum vector $\mathbf{k}$ in standard spherical coordinates. If the particles have mass, $F(\mathcal{E}, \theta, \varphi)$ can be related to the commonly used distribution function $f(\mathbf{v})$ in plasma physics via $f(\mathbf{v}) d^{3} v=F(\mathcal{E}, \theta, \varphi) d \mathcal{E} d \Omega$, where $d^{3} v=v^{2} d v d \Omega$ and $d \Omega \equiv \sin \theta d \theta d \varphi$. Hence

$$
F(\mathcal{E}, \theta, \varphi)=\frac{v}{m} f(\mathbf{v})
$$

In general, sources due to ionization involve three distinct particle populations, namely the fast particles $F^{\prime}$, consisting of incident particles that scatter after the interaction, the ejected electrons $F_{e}$, and the recoil ions $F_{i}$. Here and in the following, we assume that the target population is at rest for simplicity. The case $F^{\prime}=F_{e}$ can be treated as a special case. It is clear that the particle, momentum and energy conservation properties of the individual ionization events must reflect in the conservation properties of the source terms 
$\mathcal{S}^{\prime}, \mathcal{S}_{e}$ and $\mathcal{S}_{i}$. It is indeed possible to derive a system of source terms which satisfies all required conservation properties from a so-called kinematically complete description of the atomic fragmentation process, which completely describes the probability of all possible combinations of momentum vectors in so-called fully differential cross sections [37]. However, as it is our goal to provide practical expressions for source terms in this paper, we defer this discussion to a separate paper.

Here, we restrict ourselves to the case where the distribution function of the fast particle population $F^{\prime}\left(\mathcal{E}^{\prime}, \theta^{\prime}, \varphi^{\prime}\right)$ is - up to a scaling factor - not part of the self-consistent plasma description. This is a good approximation if (i) the production mechanism of the fast particles is (mostly) independent of the plasma dynamics, (ii) the fast particles have enough energy to leave the plasma before they thermalize, and (iii) one is mostly interested in low-frequency electrostatic turbulence, such that the only relevant interaction of the fast particles with the plasma is via ionizing collisions. This simplification applies reasonably well to plasma production schemes used in basic plasma physics experiments, such as injection of RF waves [38-40], helicon waves [25, 41, 42], or cathode-anode discharges [43, 44].

The common case of electron-impact ionization, where $F^{\prime}$ also represents an electron population, is subject to a subtlety, as the two electrons after the ionization process are in principle indistinguishable. To be consistent with our assumption that $F^{\prime}$ is mostly decoupled from the plasma, only one of the two electrons can become part of $F_{e}$, while the other must remain part of $F^{\prime}$. Fortunately, for sufficiently high incident energies $\mathcal{E}^{\prime}$, the overwhelming majority of ionization events generate one slow and one fast electron with clearly distinct energies (see figure 2 (b)). A distinction based on energy is therefore meaningful, demanding that the slower electron be part of $F_{e}$ and the faster be part of $F^{\prime}$. When integrating over the energy distribution of electrons after the ionization to determine properties of the plasma, the upper integration boundary must thus be chosen as $\left(\mathcal{E}^{\prime}-\mathcal{B}\right) / 2$, i.e. half the total available energy for the two electrons ( $\mathcal{B}$ is the binding energy). If the incident particles are not electrons, no such subtlety applies, in which case the upper integration boundary is $\mathcal{E}^{\prime}-\mathcal{B}$. Differential cross sections in atomic physics literature are generally already normalized for the applicable integration boundaries.

In the following, we formulate kinetic source terms in terms of differential cross sections and compute the self-consistent fluid source terms by carrying out the velocity-space integrals. The ejected electron population after ionization is fully described by the double- 
differential cross section

$$
\frac{d^{2} \sigma\left(\mathcal{E}^{\prime}, \mathcal{E}, \theta^{\prime \prime}\right)}{d \Omega^{\prime \prime} d \mathcal{E}}=\frac{1}{2 \pi \sin \theta^{\prime \prime}} \frac{d^{2} \sigma\left(\mathcal{E}^{\prime}, \mathcal{E}, \theta^{\prime \prime}\right)}{d \theta^{\prime \prime} d \mathcal{E}}
$$

where $\mathcal{E}^{\prime}$ is the energy of the incident particle, $\mathcal{E}$ is the energy of the ejected electron, $\theta^{\prime \prime}$ is the angle between incident and outgoing momentum vectors, given by

$$
\cos \theta^{\prime \prime} \equiv \hat{\mathbf{k}} \cdot \hat{\mathbf{k}}^{\prime}=\cos \theta \cos \theta^{\prime}+\sin \theta \sin \theta^{\prime} \cos \left(\varphi-\varphi^{\prime}\right)
$$

and $d \Omega^{\prime \prime}$ is the differential solid angle in the frame of reference aligned with the incident particle. Here, hats denote unit vectors: $\hat{\mathbf{k}} \equiv \mathbf{k} / k, \hat{\mathbf{k}}^{\prime} \equiv \mathbf{k}^{\prime} / k^{\prime}$. The target population, which can be atoms or ions, is described by a density $n_{t}$ and is assumed to be at rest. The kinetic electron source term in Eq. 1 describing particle-impact ionization is then

$$
\mathcal{S}(\mathbf{v})=\frac{m n_{t}}{v} \int d \mathcal{E}^{\prime} d \Omega^{\prime} F^{\prime}\left(\mathcal{E}^{\prime}, \theta^{\prime}, \varphi^{\prime}\right) v^{\prime} \frac{d^{2} \sigma\left(\mathcal{E}^{\prime}, \mathcal{E}, \theta^{\prime \prime}\right)}{d \Omega^{\prime \prime} d \mathcal{E}}=\frac{m n_{t} n^{\prime}}{v}\left\langle v^{\prime} \frac{d^{2} \sigma}{d \Omega^{\prime \prime} d \mathcal{E}}\right\rangle^{\prime},
$$

where $n^{\prime} \equiv \int d \mathcal{E}^{\prime} d \Omega^{\prime} F^{\prime}\left(\mathcal{E}^{\prime}, \theta^{\prime}, \varphi^{\prime}\right)$ and $v^{\prime} \equiv v^{\prime}\left(\mathcal{E}^{\prime}\right)$ are the density and the (relativistic) speed of the incident particles, respectively, and $\langle\cdot\rangle^{\prime} \equiv\left(1 / n^{\prime}\right) \int d \mathcal{E}^{\prime} d \Omega^{\prime} F^{\prime}\left(\mathcal{E}^{\prime}, \theta^{\prime}, \varphi^{\prime}\right)(\cdot)$. The factor $m / v$ results again from $d \mathcal{E}=m v d v$ and $d^{3} v=v^{2} d v d \Omega$.

A fully consistent kinetic source term for ions can in principle be inferred from the tripledifferential cross section, which, in addition to (30), is also differential in the solid angle of the scattered particle. However, this is rather complicated and will be discussed in a separate paper. Here, we only give the minimal kinetic ion source term, which is

$$
\mathcal{S}_{i}(\mathbf{v})=S_{n} \delta^{3}(\mathbf{v})
$$

This ensures that as many ions as electrons are generated, but neglects all momentum and energy sources for the ions.

The first three moments of the kinetic electron source term are:

$$
\begin{gathered}
S_{n}=\int d^{3} v \mathcal{S}=n_{t} n^{\prime} \int d \mathcal{E} d \Omega\left\langle v^{\prime} \frac{d^{2} \sigma}{d \Omega^{\prime \prime} d \mathcal{E}}\right\rangle^{\prime}, \\
\mathbf{S}_{k, L}=m \int d^{3} v \mathbf{v} \mathcal{S}=n_{t} n^{\prime} \int d \mathcal{E} d \Omega(2 m \mathcal{E})^{1 / 2} \hat{\mathbf{v}}\left\langle v^{\prime} \frac{d^{2} \sigma}{d \Omega^{\prime \prime} d \mathcal{E}}\right\rangle^{\prime}, \\
S_{\mathcal{E}, L}=\frac{m}{2} \int d^{3} v v^{2} \mathcal{S}=n_{t} n^{\prime} \int d \mathcal{E} d \Omega \mathcal{E}\left\langle v^{\prime} \frac{d^{2} \sigma}{d \Omega^{\prime \prime} d \mathcal{E}}\right\rangle^{\prime} .
\end{gathered}
$$

To calculate the integrals over the solid angle $\int_{0}^{4 \pi} d \Omega(\cdot)$, a change of variables $(\theta, \varphi) \rightarrow$ $\left(\theta^{\prime \prime}, \varphi^{\prime \prime}\right)$ is performed, corresponding to a rotation of the coordinate system such that the 
$z$ axis is aligned with $\mathbf{v}^{\prime}$. The Jacobian for this transformation is $\operatorname{det}\left(d \Omega / d \Omega^{\prime \prime}\right)=1$. Thus $\int_{0}^{4 \pi} d \Omega(\cdot)=\int_{0}^{4 \pi} d \Omega^{\prime \prime}(\cdot)=\int_{0}^{\pi} d \theta^{\prime \prime} \sin \theta^{\prime \prime} \int_{0}^{2 \pi} d \varphi^{\prime \prime}(\cdot)$. The result is

$$
\begin{aligned}
S_{n} & =n_{t} n^{\prime}\left\langle v^{\prime} \sigma\right\rangle^{\prime}, \\
\mathbf{S}_{k, L} & =n_{t} n^{\prime}\left\langle\mathbf{v}^{\prime} \sigma \kappa\right\rangle^{\prime}, \\
S_{\mathcal{E}, L} & =n_{t} n^{\prime}\left\langle v^{\prime} \sigma \epsilon\right\rangle^{\prime},
\end{aligned}
$$

where $\left\langle v^{\prime} \sigma\right\rangle^{\prime},\left\langle\mathbf{v}^{\prime} \sigma \kappa\right\rangle^{\prime}$ and $\left\langle v^{\prime} \sigma \epsilon\right\rangle^{\prime}$ are the rate coefficients for particle, momentum and energy generation, respectively. Here, we have introduced the forward momentum function $\kappa\left(\mathcal{E}^{\prime}\right)$ and the energy function $\epsilon\left(\mathcal{E}^{\prime}\right)$ of the ionization process, which are defined as

$$
\begin{aligned}
\kappa\left(\mathcal{E}^{\prime}\right) & \equiv \frac{1}{\sigma} \int d \mathcal{E}(2 m \mathcal{E})^{1 / 2} \overline{\cos \theta^{\prime \prime}} \frac{d \sigma}{d \mathcal{E}} \\
\epsilon\left(\mathcal{E}^{\prime}\right) & \equiv \frac{1}{\sigma} \int d \mathcal{E} \mathcal{E} \frac{d \sigma}{d \mathcal{E}}
\end{aligned}
$$

where

$$
\overline{\cos \theta^{\prime \prime}}\left(\mathcal{E}^{\prime}, \mathcal{E}\right) \equiv \frac{2 \pi \int_{0}^{\pi} d \theta^{\prime \prime} \sin \theta^{\prime \prime} \cos \theta^{\prime \prime} \frac{d^{2} \sigma}{d \Omega^{\prime \prime} d \mathcal{E}}}{d \sigma / d \mathcal{E}}
$$

The functions $\kappa\left(\mathcal{E}^{\prime}\right)$ and $\epsilon\left(\mathcal{E}^{\prime}\right)$ specify the average forward momentum and energy, respectively, which is passed to the ejected electron during one ionizing collision, as a function of the incident particle energy $\mathcal{E}^{\prime}$ only. They are an entirely atomic physical property and, together with $\sigma\left(\mathcal{E}^{\prime}\right)$, completely describe the interface between atomic physics and plasma physics. Knowledge of the double-differential cross section is sufficient to compute both functions. However, an important observation is that only the momentum in the forward direction, i.e. in the direction of the incident particle, is required to completely describe the plasma momentum source. This may lead to simplifications for atomic physics theory or experiments if the forward momentum function is determined directly.

It is worth mentioning that the momentum source due to the ejected electrons can be shown to vanish in many practical situations, such as photoionization or electron-impact ionization at high energies $[45,46]$, for which $\overline{\cos \theta^{\prime \prime}} \simeq 0$. This will be discussed in a separate paper. Finally, the momentum source also vanishes for arbitrary $\kappa\left(\mathcal{E}^{\prime}\right)$ if $\int d \Omega^{\prime} \hat{\mathbf{v}}^{\prime} F^{\prime}=0$. This is in particular the case for isotropic fast particle distributions. 


\begin{tabular}{|c|c|c|c|c|c|c|c|c|c|c|c|}
\hline \multirow{2}{*}{$\begin{array}{l}\text { Gas } \\
\text { Orbital }\end{array}$} & \multirow{2}{*}{$\begin{array}{c}\mathrm{H}[47] \\
1 \mathrm{~s}\end{array}$} & \multirow{2}{*}{$\begin{array}{c}\mathrm{H}_{2}[47] \\
1 \sigma\end{array}$} & \multirow{2}{*}{$\begin{array}{c}\mathrm{He}[49] \\
1 \mathrm{~s}\end{array}$} & \multicolumn{3}{|c|}{$\mathrm{Ne}[47]$} & \multicolumn{5}{|c|}{$\operatorname{Ar}[50]$} \\
\hline & & & & $2 \mathrm{p}$ & $2 \mathrm{~s}$ & $1 \mathrm{~s}$ & $3 p$ & $3 \mathrm{~s}$ & $2 p$ & $2 \mathrm{~s}$ & $1 \mathrm{~s}$ \\
\hline $\mathcal{B}[\mathrm{eV}]$ & 13.6057 & 15.9800 & 24.587 & 21.60 & 48.47 & 866.9 & 15.82 & 29.24 & 249.18 & 326.0 & 3202.9 \\
\hline$U[\mathrm{eV}]$ & 13.6057 & 15.9800 & 39.51 & 116.02 & 141.88 & 1259.1 & 78.07 & 103.5 & 651.4 & 683.1 & 4192.9 \\
\hline$N$ & 1 & 2 & 2 & 6 & 2 & 2 & 6 & 2 & 6 & 2 & 2 \\
\hline$Q$ & 0.5668 & 1.0000 & 0.8841 & 0.8213 & 0.1710 & 1.0462 & & & & & \\
\hline$K^{\mathrm{BED}}$ & 1.5657 & 1.4134 & 1.1860 & & & & & & & & \\
\hline$c_{2}$ & -0.0225 & & & & & & & & & & \\
\hline$c_{3}$ & 1.1775 & 1.1262 & 8.2401 & & & & & & & & \\
\hline$c_{4}$ & -0.4626 & 6.3982 & -10.4769 & & & & & & & & \\
\hline$c_{5}$ & 0.0890 & -7.8055 & 3.9650 & & & & & & & & \\
\hline$c_{6}$ & & 2.1440 & -0.0446 & & & & & & & & \\
\hline
\end{tabular}

TABLE I: Atomic physics parameters necessary for the BEB, BEQ and BED theories. References are given in the square brackets. The coefficients $c_{1}$ and $c_{7}$ are zero for all considered gases.

\section{THE BEB AND BED THEORIES FOR ELECTRON-IMPACT IONIZATION}

We now focus on electron-impact ionization, being the dominant plasma generation mechanism in magnetic fusion devices as well as in basic plasma physics experiments. Electronimpact ionization is extensively studied both experimentally [46] and theoretically [47] in the quantum and atomic physics communities, who have mapped out and calculated total, single-, double- and triple-differential cross sections for a wide range of atoms and molecules. The goal of this section is to make use of this knowledge to compute accurate source terms that can be used efficiently in fluid plasma physics simulations.

In particular, we will use the BEB (Binary-Encounter-Bethe) model, and its more sophisticated versions, the BEQ (for "BEB with $Q$ " in more recent publications [48]) and the BED (Binary-Encounter-Dipole) models, which are derived from first principles with some empirical adaptations in [47]. These theories remarkably achieve a completely analytical form of the single-differential cross section (SDCS), which (i) depends only on relatively well-known atomic/molecular constants, (ii) is free from adjustable parameters, (iii) is in principle applicable to any atom and sufficiently simple molecule, (iv) can be integrated analytically, and most importantly (v) has been successfully validated against experimental SDCS's for a wide range of atoms and molecules to within experimental uncertainty of typically $5-15 \%$.

According to the BEB, BEQ and BED theories, the SDCS for electron impact ionization 
results from the sum of the contributions of each orbital, each of which take the form

$$
\frac{d \sigma\left(\mathcal{E}^{\prime}, \mathcal{E}\right)}{d \mathcal{E}}=\frac{S}{\mathcal{B}(t+u+1)}\left\{-\frac{K}{t+1} f_{1}(w)+K f_{2}(w)+\ln t f_{3}(w)\right\}
$$

where $\mathcal{B}$ is the orbital binding energy, and $t \equiv \mathcal{E}^{\prime} / \mathcal{B}, w \equiv \mathcal{E} / \mathcal{B}$ and $u \equiv \mathcal{U} / \mathcal{B}$ are the normalized incident electron energy, outgoing electron energy and orbital kinetic energy, respectively. Further, $S \equiv 4 \pi a_{0}^{2} N(\mathcal{R} / \mathcal{B})^{2}$, where $a_{0} \equiv 0.52918 \times 10^{-10} \mathrm{~m}$ is the Bohr atom radius, $N$ is the electron occupation number, and $\mathcal{R} \equiv 13.6057 \mathrm{eV}$ is the Rydberg energy. The functions $f_{1}(w), f_{2}(w)$ and $f_{3}(w)$ are

$$
f_{1}(w) \equiv \frac{1}{w+1}+\frac{1}{t-w}, \quad f_{2}(w) \equiv \frac{1}{(w+1)^{2}}+\frac{1}{(t-w)^{2}},
$$

and

$$
f_{3}^{\mathrm{BEQ}}(w) \equiv Q\left(\frac{1}{(w+1)^{3}}+\frac{1}{(t-w)^{3}}\right), \quad f_{3}^{\mathrm{BED}}(w) \equiv \frac{1}{N(w+1)} \frac{d f(w)}{d w},
$$

where $d f / d w$ are the differential oscillator strengths. In the BEQ theory, these are replaced by the more generally available dipole constant $Q \equiv(2 / N) \int_{0}^{\infty} d w(w+1)^{-1} d f / d w$. The BEB theory emerges from the BEQ theory simply by setting $Q=1$. The proportionality factor $K$ is given by

$$
K^{\mathrm{BEQ}} \equiv 2-Q, \quad K^{\mathrm{BED}} \equiv 2-\frac{N_{i}}{N}
$$

where $N_{i} \equiv \int_{0}^{\infty} d w d f / d w$. In the BED theory, the differential oscillator strengths are approximated by a power series

$$
\frac{d f(w)}{d w}=\sum_{m=1}^{M} \frac{c_{m}}{(w+1)^{m}}
$$

where $M \leq 7$ and the coefficients $c_{m}$ are tabulated [47]. For easier reference, table I summarizes all necessary atomic physics parameters for the gases discussed in this paper. Reference [47] recommends to use the BED theory if $d f / d w$ is known, the BEQ theory if $Q$ is known, and the BEB theory if neither of these are available. However, it appears that the simpler BEB theory has been most widely adopted in more recent publications, presumably because the gain in accuracy does not necessarily justify the increase in complexity (figure 2 ). In addition, due to the non-symmetric form of $f_{3}(w)$ in (44), the BED theory does not look consistent with the necessary symmetry $\mathcal{E} \leftrightarrow \mathcal{E}^{\prime}-\mathcal{B}-\mathcal{E}$, which results from the fact 

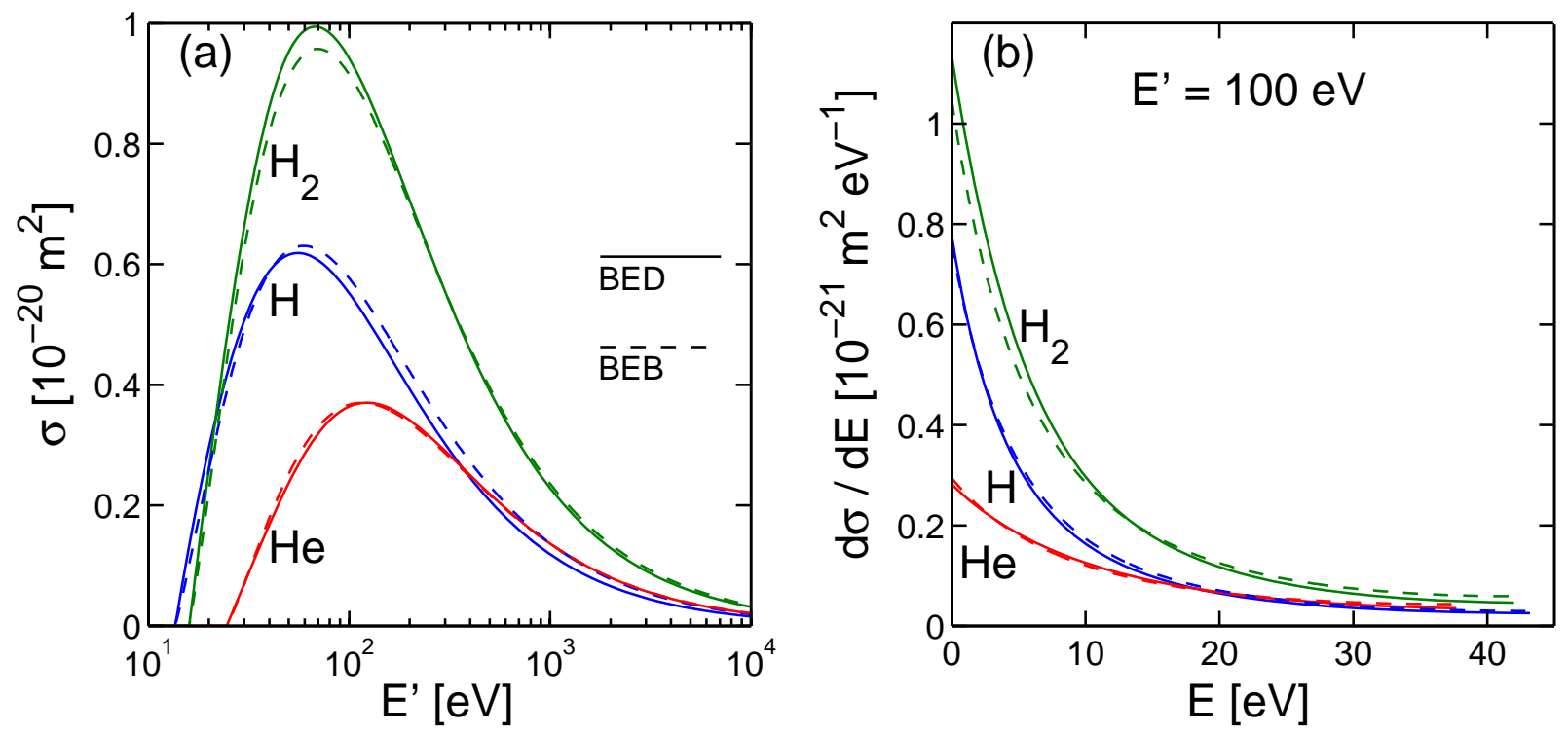

FIG. 2: Comparison between BED and BEB cross sections for $\mathrm{H}, \mathrm{H}_{2}$ and He. (a) Total cross section $\sigma\left(\mathcal{E}^{\prime}\right)$. (b) Single-differential cross section $d \sigma / d \mathcal{E}$ for $\mathcal{E}^{\prime}=100 \mathrm{eV}$.

that the incident and ejected electrons are indistinguishable after the ionization process. The interpretation of this should be clarified by the authors of the BED theory. As this paper aims at providing simple and easy-to-implement expressions for the source terms in fluid plasma simulations, we will mostly make use of the BEB theory, but perform all calculations analogously for the BED theory for completeness.

We now proceed to calculate

$$
\sigma\left(\mathcal{E}^{\prime}\right)=\int_{0}^{\frac{\mathcal{E}^{\prime}-\mathcal{B}}{2}} d \mathcal{E} \frac{d \sigma}{d \mathcal{E}}, \quad \quad \epsilon\left(\mathcal{E}^{\prime}\right)=\frac{1}{\sigma\left(\mathcal{E}^{\prime}\right)} \int_{0}^{\frac{\mathcal{E}^{\prime}-\mathcal{B}}{2}} d \mathcal{E} \mathcal{E} \frac{d \sigma}{d \mathcal{E}}
$$

where the upper integral boundary was chosen as $\left(\mathcal{E}^{\prime}-\mathcal{B}\right) / 2$ to account only for the slower electron, as discussed previously. We obtain

$$
\begin{aligned}
& \sigma\left(\mathcal{E}^{\prime}\right)=\frac{S}{t+u+1}\left\{\left(D_{0}(t)-\frac{K}{t+1}\right) \ln t+K\left(1-t^{-1}\right)\right\}, \\
& \epsilon\left(\mathcal{E}^{\prime}\right)=\frac{S}{t+u+1}\left\{\left(D_{1}(t)-K \frac{2 t+1}{t+1}\right) \ln t+3 K \ln \frac{t+1}{2}\right\} \frac{\mathcal{B}}{\sigma\left(\mathcal{E}^{\prime}\right)}
\end{aligned}
$$



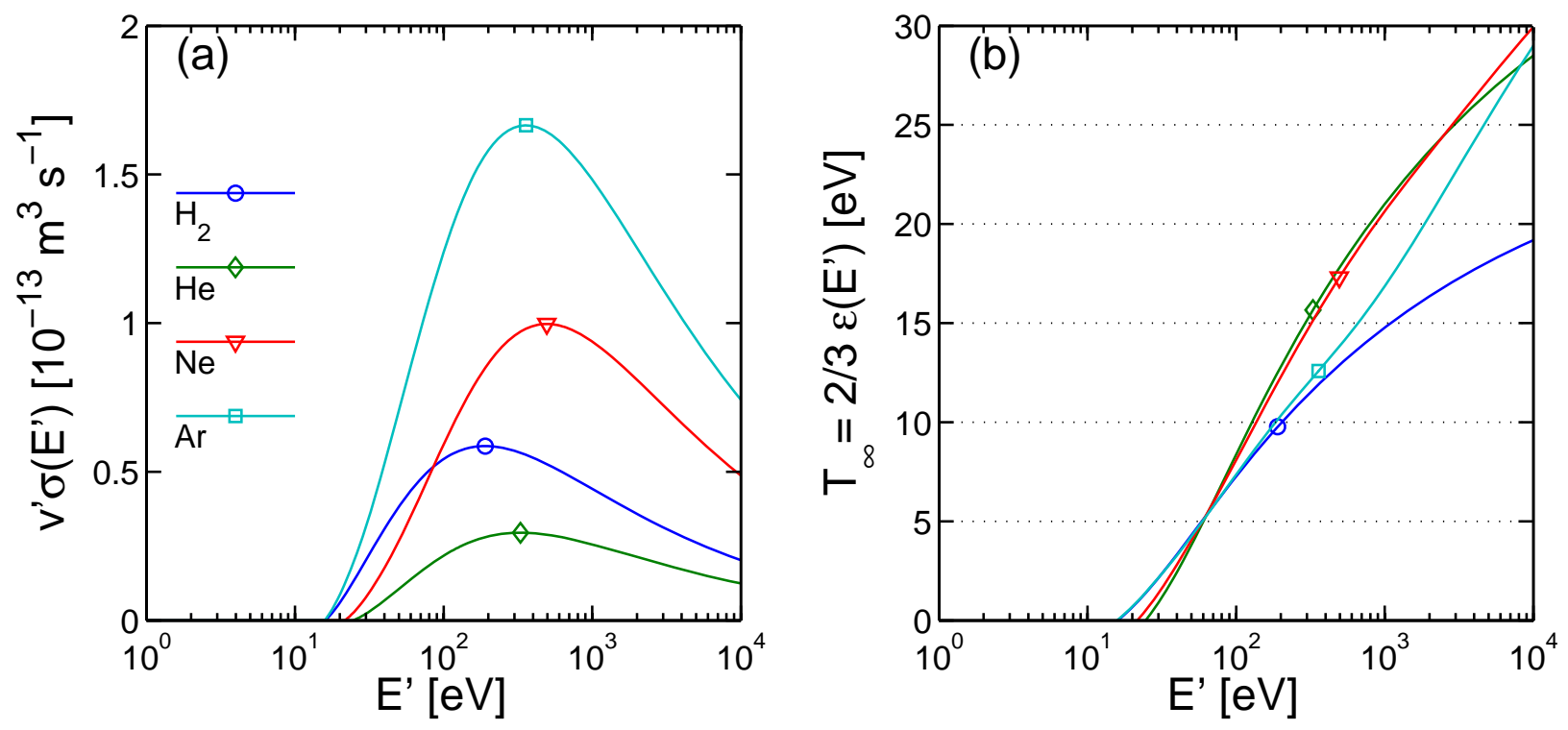

FIG. 3: Monoenergetic rate coefficients for particle generation (a) and achievable plasma electron temperature (b) as a function of the incident electron energy $\mathcal{E}^{\prime}$ for $\mathrm{H}_{2}, \mathrm{He}, \mathrm{Ne}$ and $\mathrm{Ar}$ in the BEB model. Markers indicate values at the peak ionization efficiency.

where $D_{n}(t) \equiv \int_{0}^{(t-1) / 2} d w w^{n} f_{3}(w)$ is

$$
\begin{aligned}
& D_{0}^{\mathrm{BEQ}}(t)=\frac{Q}{2}\left(1-t^{-2}\right), \\
& D_{1}^{\mathrm{BEQ}}(t)=\frac{Q}{2} \frac{(t-1)^{2}}{t(t+1)},
\end{aligned}
$$

and

$$
\begin{aligned}
& D_{0}^{\mathrm{BED}}(t)=\frac{1}{N} \sum_{m=1}^{M} c_{m}\left(1-\left(\frac{2}{t+1}\right)^{m}\right), \\
& D_{1}^{\mathrm{BED}}(t)=\frac{1}{N}\left\{\sum_{m=1}^{M-1} \frac{c_{m+1}+c_{m}}{m}\left(1-\left(\frac{2}{t+1}\right)^{m}\right)-\frac{c_{M}}{M}\left(1-\left(\frac{2}{t+1}\right)^{M}\right)-c_{1} \ln \frac{2}{t+1}\right\} .
\end{aligned}
$$

The results from equation (48), in the form of $v^{\prime} \sigma\left(\mathcal{E}^{\prime}\right)$ and $\frac{2}{3} \epsilon\left(\mathcal{E}^{\prime}\right)$, are shown in figure 3 for the BEB model for $\mathrm{H}_{2}, \mathrm{He}, \mathrm{Ne}$ and Ar.

\section{A. Monoenergetic population of fast electrons}

In the case of plasma production by hot cathode and extraction anode, such as used in the linear device LAPD [51], the fast electron population is well described by a monoenergetic 

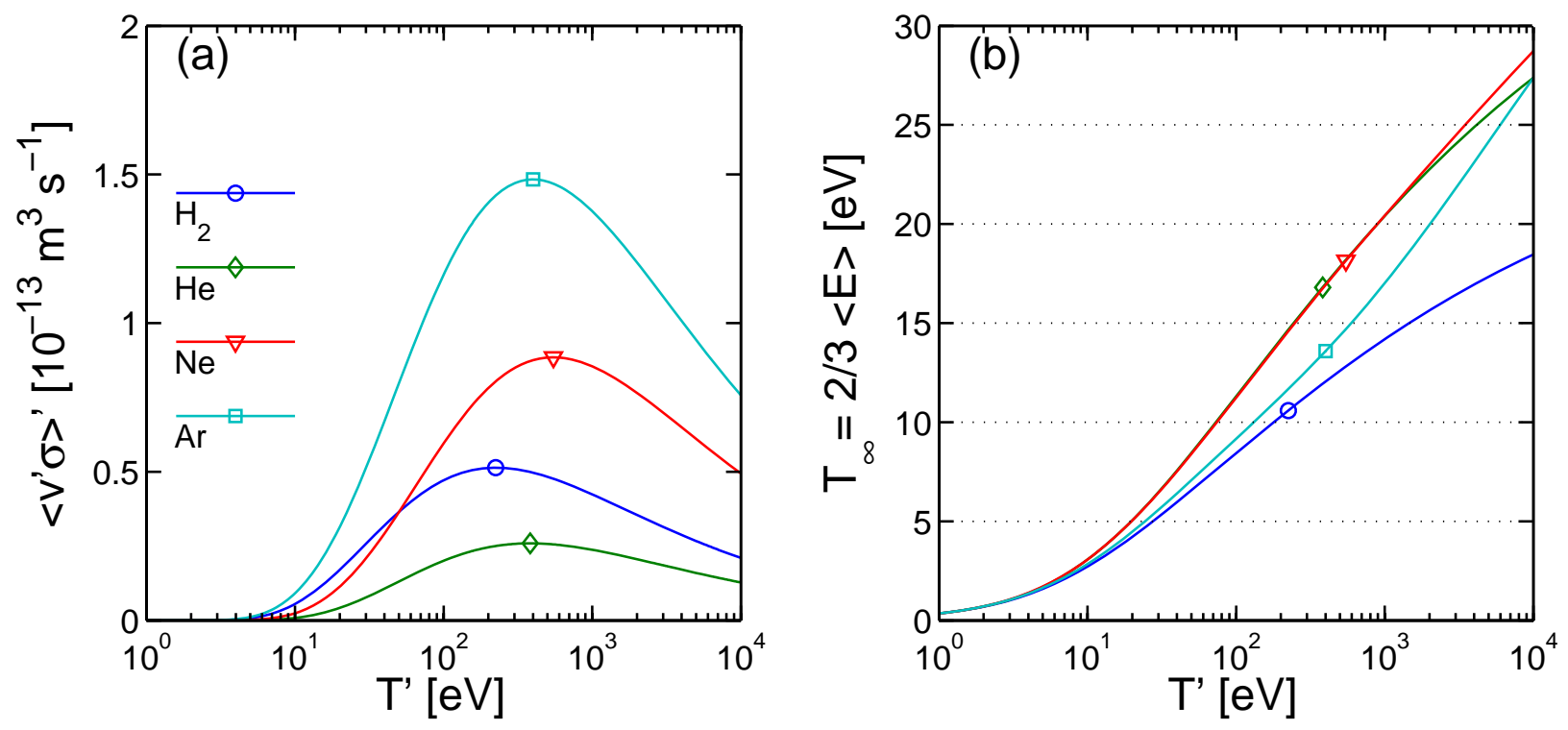

FIG. 4: Maxwellian-averaged rate coefficients for particle generation (a) and achievable plasma electron temperature (b) as a function of the fast electron temperature $T^{\prime}$ for $\mathrm{H}_{2}$, He, $\mathrm{Ne}$ and $\mathrm{Ar}$ in the BEB model. Markers indicate values at the peak ionization efficiency.

directed beam

$$
F^{\prime}\left(\mathcal{E}^{\prime}, \theta^{\prime}, \varphi^{\prime}\right)=\frac{n^{\prime}}{2 \pi \sin \theta^{\prime}} \delta\left(\mathcal{E}^{\prime}-e V^{\prime}\right) \delta\left(\theta^{\prime}\right),
$$

where $V^{\prime}$ is the extraction voltage. We obtain

$$
\begin{aligned}
S_{n} & =n_{t} n^{\prime} \sqrt{\frac{2 e V^{\prime}}{m}} \sigma\left(e V^{\prime}\right), \\
\langle\mathbf{k}\rangle & =\kappa\left(e V^{\prime}\right) \hat{\mathbf{z}}, \\
\langle\mathcal{E}\rangle & =\epsilon\left(e V^{\prime}\right) .
\end{aligned}
$$

The maximum plasma electron temperature as a function of the extraction voltage is thus given by

$$
T_{\infty}\left(V^{\prime}\right)=\frac{2}{3}\left(\epsilon\left(e V^{\prime}\right)-\frac{\kappa^{2}\left(e V^{\prime}\right)}{2 m}\right) \simeq \frac{2}{3} \epsilon\left(e V^{\prime}\right),
$$

where $\epsilon\left(e V^{\prime}\right)$ is given by (48b) and shown in figure 3(b). Measurement of the electron temperature in LAPD as a function of the extraction voltage would constitute a good experimental test of the proposed source terms. 


\section{B. Maxwellian population of fast electrons}

In the case that the electron population responsible for ionization is described by an isotropic Maxwellian with temperature $T^{\prime}$ :

$$
F^{\prime}\left(\mathcal{E}^{\prime}, \theta^{\prime}, \varphi^{\prime}\right)=\frac{n^{\prime}}{4 \pi} \frac{2}{\sqrt{\pi}}\left(\frac{\mathcal{E}^{\prime}}{T^{\prime}}\right)^{1 / 2} \exp \left(-\frac{\mathcal{E}^{\prime}}{T^{\prime}}\right) \frac{1}{T^{\prime}}
$$

we have

$$
\begin{aligned}
S_{n} & =n_{t} n^{\prime} \frac{2 \sqrt{2}}{\sqrt{\pi m T^{\prime 3}}} \int d \mathcal{E}^{\prime} \mathcal{E}^{\prime} \exp \left(-\frac{\mathcal{E}^{\prime}}{T^{\prime}}\right) \sigma\left(\mathcal{E}^{\prime}\right), \\
\langle\mathbf{k}\rangle & =0, \\
\langle\mathcal{E}\rangle & =\frac{\int d \mathcal{E}^{\prime} \mathcal{E}^{\prime} \exp \left(-\frac{\mathcal{E}^{\prime}}{T^{\prime}}\right) \sigma \epsilon\left(\mathcal{E}^{\prime}\right)}{\int d \mathcal{E}^{\prime} \mathcal{E}^{\prime} \exp \left(-\frac{\mathcal{E}^{\prime}}{T^{\prime}}\right) \sigma\left(\mathcal{E}^{\prime}\right)} .
\end{aligned}
$$

The results are shown in figure 4, which will be discussed and compared to the monoenergetic case (figure 3 ) in the following.

\section{DISCUSSION}

The most obvious conclusion from figures 3 and 4 is that it is inherently difficult to influence the plasma electron temperature by changing the parameters of the source, which ultimately requires changing the energy of the fast electrons. As a rough guideline, to achieve a $50 \%$ increase in $T_{e}$, the primary electron energy would have to be increased by an order of magnitude. In addition, such a change would have a somewhat stronger effect on the plasma density, which in first approximation is proportional to the rate coefficients $\left\langle v^{\prime} \sigma\right\rangle^{\prime}$. Experimentalists therefore - implicitly or explicitly - optimize their setup to operate at a working point close to the maximum ionization efficiency, which also has the advantage to maximize the probability of a breakdown. We therefore argue that, for the common case where the primary electron energies are unknown, the most suitable assumption for simulation source parameters $\mathcal{E}^{\prime}$ or $T^{\prime}$ is one that maximizes the ionization efficiency $\left\langle v^{\prime} \sigma\right\rangle^{\prime}$. The weak dependence of $\sigma\left(\mathcal{E}^{\prime}\right)$ and $\epsilon\left(\mathcal{E}^{\prime}\right)$ on $\mathcal{E}^{\prime}$ makes this assumption suitable for a wide range of conditions.

The values at the peak ionization efficiency are highlighted in figures 3 and 4 , and reproduced in table II. First, we note that the results are rather insensitive to the actual 


\begin{tabular}{l|ccc|ccc}
\hline \hline & \multicolumn{3}{|c|}{ Monoenergetic } & \multicolumn{3}{c}{ Maxwellian } \\
& $\mathcal{E}_{\text {peak }}^{\prime}[\mathrm{eV}]$ & $v^{\prime} \sigma_{\text {peak }}\left[10^{-13} \mathrm{~m}^{3} \mathrm{~s}^{-1}\right]$ & $T_{\infty, \text { peak }}[\mathrm{eV}]$ & $T_{\text {peak }}^{\prime}[\mathrm{eV}]$ & $\left\langle v^{\prime} \sigma\right\rangle_{\text {peak }}^{\prime}\left[10^{-13} \mathrm{~m}^{3} \mathrm{~s}^{-1}\right]$ & $T_{\infty, \text { peak }}[\mathrm{eV}]$ \\
\hline $\mathrm{H}_{2}$ & 190 & 0.59 & 9.8 & 224 & 0.51 & 10.6 \\
$\mathrm{He}$ & 329 & 0.30 & 15.7 & 382 & 0.26 & 16.8 \\
$\mathrm{Ne}$ & 495 & 1.00 & 17.3 & 548 & 0.88 & 18.1 \\
$\mathrm{Ar}$ & 358 & 1.67 & 12.6 & 401 & 1.48 & 13.6 \\
\hline \hline
\end{tabular}

TABLE II: Fast electron energy $\mathcal{E}_{\text {peak }}^{\prime}$, rate coefficients $\left\langle v^{\prime} \sigma\right\rangle_{\text {peak }}^{\prime}$, and maximum achievable electron temperature $T_{\infty, \text { peak }}$, at the peak ionization efficiency, compared for the monoenergetic (figure 3 ) and Maxwellian cases (figure 4).

shape of the fast electron distribution, as the parameters characterizing the peak differ by only 10-20\% between the monoenergetic and Maxwellian cases. The optimum fast electron energies, which should result in the highest plasma densities, are in the range of $200 \mathrm{eV}$ for hydrogen and 300-550 eV for the noble gases. The trends of increasing $\left\langle v^{\prime} \sigma\right\rangle_{\text {peak }}^{\prime}$ from helium to neon to argon agree with experimentally observed trends in the plasma density in the TJ-K [25] and TORPEX devices [52], but the prediction of a higher plasma density in $\mathrm{H}_{2}$ than in helium is at odds with the experimental observations [33, 52, 53]. This could be due to several factors, including (i) the fact that both non-dissociating $\left(\mathrm{H}_{2}+e^{-} \rightarrow \mathrm{H}_{2}^{+}+2 e^{-}\right)$and dissociating $\left(\mathrm{H}_{2}+e^{-} \rightarrow \mathrm{H}+\mathrm{H}^{+}+2 e^{-}\right)$ionization events exist, which the theories discussed here are not able to distinguish, (ii) the strong chemical activity of hydrogen, and (iii) the fact that hydrogen is subject to larger sheath losses due to the smaller ion mass. Ultimately, sources, transport and sinks must be simultaneously understood for a rigorous comparison with experimental profiles, which makes this task so challenging.

The biggest success of the presented source terms is the explanation of the maximum plasma electron temperatures achieved in basic plasma physics experiments, including the trends across different gases, which are compiled in table III for a wide range of devices. A comprehensive database of different gases has been gathered on the torsatron TJ-K for different plasma production schemes $[25,53,54]$. In inductively coupled hydrogen discharges, a maximum electron temperature of $12 \mathrm{eV}$ was observed [53]. In helicon discharges, the temperatures in helium did not exceed $23 \mathrm{eV}$, with most observations less than $18 \mathrm{eV}$, while the temperature in argon was found to be less than $10 \mathrm{eV}$ [25]. In RF discharges, the maximum values were $13 \mathrm{eV}$ for hydrogen, $19 \mathrm{eV}$ for helium, with most observations less than $13 \mathrm{eV}$, and $12 \mathrm{eV}$ for argon [39]. These temperatures are in good quantitative agreement with 


\begin{tabular}{|c|c|c|c|c|c|c|c|c|c|c|c|}
\hline & \multicolumn{3}{|c|}{ Linear devices } & \multicolumn{4}{|c|}{ Simple magnetized tori } & \multicolumn{3}{|c|}{ Torsatron } & \multirow{3}{*}{$\begin{array}{l}T_{\infty} \text {,peak } \\
\text { Maxw. }\end{array}$} \\
\hline & CSDX & VINETA & LAPD & BETA & BLAAM & ANN & TORPEX & & TJ-K & & \\
\hline & Helic. [41] & Helic. [42] & Cath. [44] & $\mathrm{RF}[38]$ & Filam. [43] & $\mathrm{RF}[55]$ & $\mathrm{RF}[52]$ & Induct. [53] & Helic. [25] & $\mathrm{RF}[39]$ & \\
\hline $\mathrm{H}_{2}$ & & & & 7 & 7.5 & 4 & 8 & 12 & & 13 & 10.6 \\
\hline $\mathrm{He}$ & & & 8 & & & 9 & 14 & & $18(23)$ & $13(19)$ & 16.8 \\
\hline $\mathrm{Ne}$ & & & & & & & 12 & & & & 18.1 \\
\hline $\mathrm{Ar}$ & 3 & 3.5 & & & & 6 & 7 & & 10 & 12 & 13.6 \\
\hline
\end{tabular}

TABLE III: Compilation of the maximum electron temperatures in $\mathrm{eV}$ reported from different basic plasma physics experiments for different plasma production schemes. References are given in the square brackets. Values in parentheses are statistical outliers. The maximum achievable electron temperature derived from the source terms (last column) explains the experimentally reached temperatures and correctly captures the trends across different gases.

the predictions from the BEB theory $\left(\mathrm{H}_{2}: 10.6 \mathrm{eV}\right.$; He: $16.8 \mathrm{eV}$; Ar: $\left.13.6 \mathrm{eV}\right)$. In particular, our source terms correctly capture the fact that helium plasmas are systematically about 30$100 \%$ hotter than hydrogen and argon plasmas, which is also reported from RF discharges in the simple magnetized tori BLAAMANN ( $\mathrm{H}_{2}: 4 \mathrm{eV}$; He: $9 \mathrm{eV}$; Ar: $6 \mathrm{eV}$ [55]) and TORPEX $\left(\mathrm{H}_{2}: 8 \mathrm{eV}\right.$; He: $14 \mathrm{eV}$; Ar: $7 \mathrm{eV}$ [52]). Electron temperatures for neon are found similar to helium in TORPEX (He: $14 \mathrm{eV}$; Ne: $12 \mathrm{eV}$ [52]), which is also consistent with the BEB theory prediction (He: $16.8 \mathrm{eV}$; Ne: $18.1 \mathrm{eV}$ ).

This kind of agreement is remarkable, given that neither any plasma transport and loss mechanisms have yet been considered, nor any knowledge of the primary electron energies has been available. This highlights the excellent potential of the proposed source terms to significantly improve the realism of simulations of basic plasma physics experiments and tokamak SOLs.

\section{A. Implications for TORPEX 'H-mode' simulations}

In the following, we discuss the implications of this work for two-dimensional interchange turbulence simulations [30], which predict an 'H-mode' like regime in TORPEX for long parallel connection lengths and high temperature source strengths. Experiments in which the connection length was scanned over the full geometrically possible range, by using the vertical magnetic field $B_{z}$ as the control parameter [56], did not lead to the identification of such a regime [33]. Subsequent investigations showed problems in relating the temperature source 


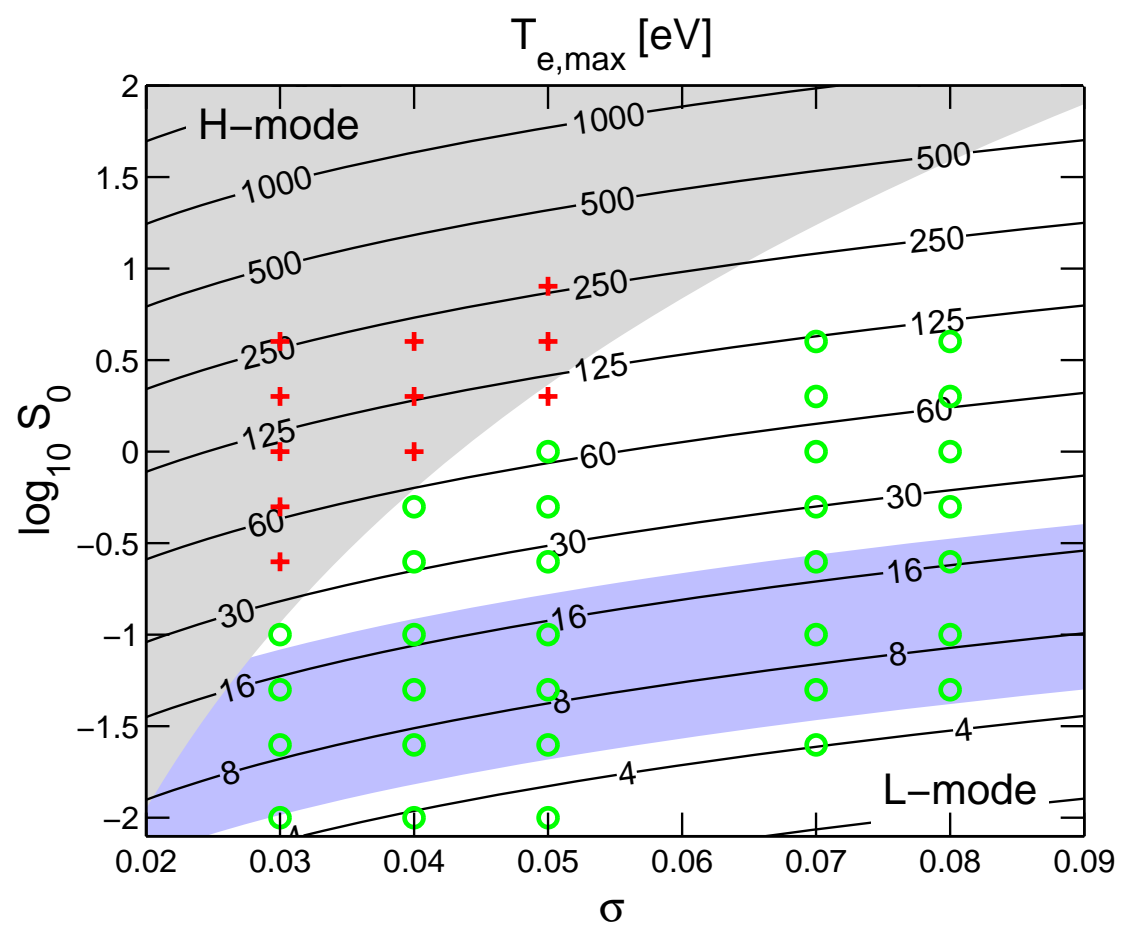

FIG. 5: Maximum time-average electron temperature achieved by 2D TORPEX simulations in [30] (from equation (64)). Simulations falling in the 'H-mode' (red crosses) and 'L-mode' (green circles) regimes are indicated (from figure 4 in [30]). Temperature regimes consistent with the source terms presented in this work and with experimental observations are highlighted in blue. Overlap with the 'H-mode' regime calculated by equation (63) is restricted to a small region at small $\sigma$, not comprising actual simulation runs. This region has been found experimentally to be characterized by $3 \mathrm{D}$ dynamics [33].

strength used in the simulation to actual physical observables that could be experimentally measured or estimated. This motivated in part the detailed investigation of the correct formulation of source terms presented in this paper.

In [30], a normalized temperature source of the form

$$
\hat{S}_{T}=\hat{S}_{0}\left\{S_{\mathrm{UH}} \exp \left(-\frac{\left(x-x_{\mathrm{UH}}\right)^{2}}{\lambda_{\mathrm{UH}}^{2}}\right)+S_{\mathrm{EC}} \exp \left(-\frac{\left(x-x_{\mathrm{EC}}\right)^{2}}{\lambda_{\mathrm{EC}}^{2}}\right)\right\}
$$

was assumed, where $S_{\mathrm{UH}}, x_{\mathrm{UH}}, \lambda_{\mathrm{UH}}, S_{\mathrm{EC}}, x_{\mathrm{EC}}$ and $\lambda_{\mathrm{EC}}$ are geometry related parameters, and $\hat{S}_{0}$ is a constant source strength, which was used as an independent variable in the simulation setup. Reference [30] found empirically that the transition from 'L-mode' to 
'H-mode' occurs at

$$
\frac{\gamma_{0}}{v_{\mathbf{E} \times \mathbf{B}}^{\prime}} \simeq \frac{2 \Delta^{3 / 2}}{\chi \Lambda \rho_{s 0} R^{1 / 2}}\left(\frac{T_{e 0}}{T_{e, \max }}\right)^{1 / 2} \simeq 0.5,
$$

where $\gamma_{0} / v_{\mathbf{E} \times \mathbf{B}}^{\prime}$ is the ratio between interchange growth rate and $\mathbf{E} \times \mathbf{B}$ shearing rate, $R=1$ $\mathrm{m}$ is the major radius, $\Delta \equiv 2 \pi r B_{z} / B_{\varphi}$ is the field-line return distance used as an independent variable, $\Lambda \equiv \frac{1}{2} \ln \left(m_{i} /\left(2 \pi m_{e}\right)\right)=3, T_{e, \max }$ is the maximum time-average electron temperature achieved in the simulation, and $\chi \simeq 2.5$ is an empirical parameter related to the maximum shear flow allowed by the Kelvin-Helmholtz instability. The normalization factors were $\rho_{s 0}=5 \mathrm{~mm}$ and $T_{e 0}=10.02 \mathrm{eV}$ for a toroidal magnetic field of $B_{\varphi}=0.076 \mathrm{~T}$. It is clear from (63), as well as from the structure of the equations, which involve terms proportional to $T_{e}^{\{0,1 / 2,1,3 / 2,2\}}$, that the absolute temperature level is the determining factor in the observed 'L-H transition', not the source strength $\hat{S}_{0}$, which was merely used to express equation (63) in terms of the independent variables of the simulation setup. Using global energy balance, reference [30] obtained

$$
T_{e, \max } \simeq T_{e 0}\left(\frac{\lambda_{\text {eff }} \hat{S}_{0}}{\sigma\left(\frac{2}{3} \xi x_{S}+\frac{4}{9} L_{T}\right)}\right)^{2 / 3},
$$

where $\lambda_{\text {eff }} \equiv \sqrt{\pi}\left(S_{\mathrm{UH}} \lambda_{\mathrm{UH}}+S_{\mathrm{EC}} \lambda_{\mathrm{EC}}\right)=10 \sqrt{\pi} \rho_{s 0}, \sigma \equiv \Delta /\left(2 \pi L_{v}\right), L_{v}=64 \rho_{s 0}$ is the torus height, $x_{S}=36 \rho_{s 0}$ is the effective source center, $\xi \simeq 0.5$ is a shaping factor, and $L_{T}$ is the temperature gradient scale length in the exponential part of the profile. Note the different meaning of the symbol $\sigma$ in the notation of reference [30].

In figure 5, equation (64) is used to reconstruct the maximum electron temperatures in physical units for the simulation runs in [30]. An unphysical rise of $T_{e, \max }$ up to $250 \mathrm{eV}$ is observed as $\hat{S}_{0}$ is scanned. This is confirmed independently by the 'H-mode' example profile in figure 1 in reference [30], from which $T_{e, \max } \simeq 230 \mathrm{eV}$ is deduced.

It is clear that this problem is related to the fact that the temperature source $S_{T}$ in the simulation is not of the correct physical form given by equations (20) and (21), which, as we have shown in this work, guarantees that the temperature stays within realistic boundaries. We have also shown that $S_{T}$ cannot be a constant, especially when saturation of the temperature source occurs at $T_{e, \max } \sim \frac{2}{3}\langle\mathcal{E}\rangle$, in which case $S_{T}$ fluctuates around zero. Even in cases where the simulation achieves realistic values of $T_{e, \max }$, the existence of a constant $S_{T}$ in the equations is problematic, as it may drive spurious DC flows. Of course, it is difficult to predict how exactly the simulation would behave with the source terms derived in this 
paper, but it seems plausible that the parameter regime of the 'H-mode' would be much reduced or even eliminated, which would be consistent with the experimental observations [33]. The strong role of the allegedly independent variable $\hat{S}_{0}$ would be replaced by a much weaker role of the fast electron energy distribution, which remains to be estimated from first principles or experimental data.

\section{SUMMARY AND CONCLUSIONS}

We have derived expressions for particle, momentum and energy sources that are suitable for direct implementation in fluid plasma simulation codes. It was shown that the interface between atomic physics and plasma physics is completely described by three scalar functions of the incident particle energy, namely the total cross section $\sigma\left(\mathcal{E}^{\prime}\right)$, the forward momentum function $\kappa\left(\mathcal{E}^{\prime}\right)$ and the energy function $\epsilon\left(\mathcal{E}^{\prime}\right)$, which are properties of the atomic physics differential cross sections. The BEB and BED theories for electron-impact ionization were used to capture the atomic physics of different gases in simple yet accurate analytical forms, making the resulting source terms practical for use in fast-timescale turbulence codes. This represents the first time that differential cross sections from atomic physics research have been applied in plasma turbulence research. It was shown that this leads to the explanation of the observations in basic plasma physics experiments regarding achievable electron temperature regimes as well as density and temperature trends across different gases.

For applications aimed at the direct quantitative comparison with the experiment, it is concluded that the self-consistent formulation of the sources and the correct atomic physical description of the working gas is just as important as the correct description of sinks, such as ion-mass dependent sheath losses. We recommend to use the source terms derived from the BEB theory for all simulations of devices that are dominated by volume ionization sources, which includes linear devices, simple magnetized tori, and torsatrons. Although a strong role of the fast electron energy distribution $F^{\prime}\left(\mathcal{E}^{\prime}\right)$ is not expected, a better understanding of the electron acceleration processes in the various plasma production schemes, such as helicon or $\mathrm{RF}$, would be desirable to improve the estimations of $F^{\prime}\left(\mathcal{E}^{\prime}\right)$ as a function of external control parameters such as the neutral gas density and the injected power. 


\section{Acknowledgments}

We thank T. A. Carter, A. Fasoli and D. Stotler for valuable discussions. This work was performed under US Department of Energy DOE Grant No. DE-FG02-06ER54871.

[1] R. E. Waltz, J. Candy, F. L. Hinton, et al. Nucl. Fusion, 45:741, 2005.

[2] S. Jolliet, B. F. McMillan, T. Vernay, et al. Phys. Plasmas, 16:052307, 2009.

[3] Y. Idomura, H. Urano, N. Aiba, and S. Tokuda. Nucl. Fusion, 49:065029, 2009.

[4] C. S. Chang, S. Ku, and H. Weitzner. Phys. Plasmas, 11:2649, 2004.

[5] G. Pereverzev et al. IPP report, 5:42, 1991.

[6] W. W. Pfeiffer, R. H. Davidson, R. W. Miller, and R. E. Waltz. General Atomics Report, GA-A16178, 1980.

[7] R. V. Budny, M. G. Bell, A. C. Janos, et al. Nucl. Fusion, 35(12):1497, 1995.

[8] D. Reiter. J. Nucl. Mater., 196-198:80, 1992.

[9] A. Taroni, G. Corrigan, G. Redford, et al. Contrib. Plasma Phys., 32(3-4), 1992.

[10] T. D. Rognlien, P. N. Brown, R. B. Campbell, et al. Contrib. Plasma Phys., 34(2-3), 1994.

[11] A. H. Kritz, G. Bateman, J. Kinsey, et al. Computer Phys. Comm., 164:108, 1997.

[12] K. Matsuda. IEEE Transactions on Plasma Science, 17:6, 1989.

[13] R. Cohen. Phys. Fluids, 30(8):2442, 1987.

[14] A. Pankin, D. McCune, R. Andre, et al. Computer Phys. Comm., 159:157, 2004.

[15] R. J. Goldston, D. C. McCune, H. H. Towner, et al. J. Comput. Phys., 43:61, 1981.

[16] D. Stotler and C. Karney. Contrib. Plasma Phys., 34(2-3):392, 1994.

[17] X. Q. Xu and R. H. Cohen. Contrib. Plasma Phys., 38(1-2), 1998.

[18] O. E. Garcia, V. Naulin, A. Nielsen, and J. J. Rasmussen. Phys. Rev. Lett., 92:165003, 2004.

[19] N. Krause, C. Lechte, J. Stöber, U. Stroth, et al. Rev. Sci. Instrum., 73(10):3474-3481, 2002.

[20] G. R. Tynan, M. J. Burin, C. Holland, et al. Phys. Plasmas, 11:5195, 2004.

[21] C. Schröder, O. Grulke, T. Klinger, et al. Phys. Plasmas, 12(4):042103, 2005.

[22] A. Fasoli, B. Labit, M. McGrath, et al. Phys. Plasmas, 13:055902, 2006.

[23] J. C. Perez, W. Horton, K. Gentle, et al. Phys. Plasmas, 13(3):032101, 2006.

[24] S. Niedner, B. D. Scott, and U. Stroth. Plasma Phys. Control. Fusion, 44:397-408, 2002. 
[25] C. Lechte, S. Niedner, and U. Stroth. New Jour. Phys., 4:34.1-34.16, 2002.

[26] C. Holland, G. R. Tynan, J. H. Yu, et al. Plasma Phys. Control. Fusion, 49:A109, 2007.

[27] V. Naulin, T. Windisch, and O. Grulke. Phys. Plasmas, 15:012307, 2008.

[28] G. N. Kervalishvili, R. Kleiber, R. Schneider, et al. Contrib. Plasma Phys., 48(1-3):32-36, 2008.

[29] T. Windisch, O. Grulke, R. Schneider, and G. N. Kervalishvili. Contrib. Plasma Phys., 48:58, 2008.

[30] P. Ricci, B. N. Rogers, and S. Brunner. Phys. Rev. Lett., 100:225002, 2008.

[31] P. Ricci, C. Theiler, A. Fasoli, et al. Phys. Plasmas, 16:055703, 2009.

[32] S. H. Müller, A. Diallo, A. Fasoli, et al. Phys. Plasmas, 14:110704, 2007.

[33] S. H. Müller, C. Theiler, A. Fasoli, et al. Plasma Phys. Control. Fusion, 51:055020, 2009.

[34] Chapter 4 ITER Physics Basis. Nucl. Fusion, 39(12):2391, 1999.

[35] A. N. Simakov and P. J. Catto. Phys. Plasmas, 10:4744, 2003.

[36] A. N. Simakov and P. J. Catto. Contrib. Plasma Phys., 44:83-94, 2004.

[37] M. Schulz, R. Moshammer, D. Fischer, et al. Nucl. Instrum. and Methods in Phys. Research $B, 267: 187,2009$.

[38] P. K. Sharma, J. P. Singh, and D. Bora. Plasma Phys. Control. Fusion, 39:1669, 1997.

[39] U. Stroth, F. Greiner, C. Lechte, et al. Phys. Plasmas, 11(5):2558, 2004.

[40] M. Podestà, A. Fasoli, B. Labit, et al. Plasma Phys. Control. Fusion, 48:1053-1062, 2006.

[41] M. J. Burin, G. R. Tynan, G. Y. Antar, et al. Phys. Plasmas, 12:052320, 2005.

[42] O. Grulke, S. Ullrich, T. Windisch, and T. Klinger. Plasma Phys. Control. Fusion, 49:B247, 2007.

[43] K. Rypdal, E. Gronvoll, F. Oynes, et al. Plasma Phys. Control. Fusion, 34:1099, 1994.

[44] T. A. Carter and J. E. Maggs. Phys. Plasmas, 16:012304, 2009.

[45] Y.-K. Kim. Phys. Rev. A, 28(2):656, 1983.

[46] H. Ehrhardt, K. Jung, G. Knoth, and P. Schlemmer. Z. Phys. D, 1:3-32, 1985.

[47] Y.-K. Kim and M. E. Rudd. Phys. Rev. A, 50:3954, 1994.

[48] Y.-K. Kim, J. P. Santos, and F. Parente. Phys. Rev. A, 62:052710, 2000.

[49] Y.-K. Kim, W. R. Johnson, and M. E. Rudd. Phys. Rev. A, 61:034702, 2000.

[50] M. E. Rudd, Y.-K. Kim, D. H. Madison, and T. J. Gay. Rev. Mod. Phys., 64(2):441, 1992.

[51] W. Gekelman, H. Pfister, Z. Lucky, et al. Rev. Sci. Instrum., 62(12):2875-2883, 1991. 
[52] C. Theiler, I. Furno, P. Ricci, et al. Phys. Rev. Lett., 103:065001, 2009.

[53] N. Krause. Untersuchung von Helikonwellen zur Plasmaheizung im Torsatron TJ-K. Ph.D. Thesis (in German), Christan Albrechts University of Kiel, Germany, 2003.

[54] M. Ramisch, N. Mahdizadeh, U. Stroth, et al. Phys. Plasmas, 12:032504, 2005.

[55] K. Rypdal, A. Fredriksen, O. M. Olsen, and K. G. Hellblom. Phys. Plasmas, 4(5):1468, 1997.

[56] S. H. Müller, A. Fasoli, B. Labit, et al. Phys. Plasmas, 12(9):090906, 2005. 